\title{
Beneficial Effects of a Flavonoid Fraction of Herba Epimedii on Bone Metabolism in Ovariectomized Rats
}

Authors

Affiliations
Bing-jie Zhao ${ }^{1 *}$, Jing Wang ${ }^{2 *}$, Jie Song ${ }^{2}$, Chun-fei Wang², Jun-fei Gu² ${ }^{2}$ Jia-rui Yuan ${ }^{2}$, Li Zhang ${ }^{2}$, Jun Jiang ${ }^{2}$, Liang Feng ${ }^{2}$, Xiao-bin Jia ${ }^{2}$

${ }^{1}$ Affiliated Hospital of Integrated Traditional Chinese and Western Medicine, Nanjing University of Chinese Medicine, Jiangsu, Nanjing, P.R. China

${ }^{2}$ Key Laboratory of New Drug Delivery Systems of Chinese Materia Medica, Jiangsu Provincial Academy of Chinese Medicine, jiangsu, Nanjing, P. R. China

\author{
Key words \\ - Herba Epimedii \\ - Berberidaceae \\ - flavonoid fraction \\ - antiosteoporotic activity \\ - ovariectomized rat
}

received June 4, 2015

revised Sept. 6, 2015

accepted October 20, 2015

\section{Bibliography}

DOI http://dx.doi.org/

10.1055/s-0035-1558294

Published online January 29,

2016

Planta Med 2016; 82: 322-329

(c) Georg Thieme Verlag KG

Stuttgart · New York ·

ISSN 0032-0943

\section{Correspondence}

Prof. Xiao-bin Jia

Affiliated Hospital

of Integrated Traditional

Chinese and Western Medicine

Nanjing University

of Chinese Medicine

Shizi Street 100\#,

Hongshan road

jiangsu, Nanjing, 210028

P. R. China

Phone: + 862585637809

Fax: + 862585502829

jxiaobin2005@hotmail.com

\section{Correspondence}

\section{Liang Feng}

Key Laboratory of New Drug

Delivery Systems of Chinese

Materia Medica

jiangsu Provincial Academy

of Chinese Medicine

Shizi Street 100\#,

Hongshan road

jiangsu, Nanjing, 210028

P. R. China

Phone: + 862552362115

Fax: + 862585502829

wenmoxiushi@163.com

\section{Abstract \\ $\nabla$}

A flavonoid fraction of Herba Epimedii, including eight flavonoid glycoside compounds, epimedoside $A$, ikarisoside $F$, baohuoside II, sagittatoside A, sagittatoside B, 7-O-rhamnosyl icariside II, 2"O-rhamnosyl icariside II, and baohuoside I, was isolated and prepared from the leaves of Herba Epimedii. This study was conducted to assess the potential effect of the flavonoid fraction of Herba Epimedii on osteoporosis in ovariectomized rats. Rats received repeated administration of a vehicle (ovariectomized), the flavonoid fraction of Herba Epimedii $(7.5,15,30 \mathrm{mg} / \mathrm{kg} / \mathrm{d})$, and ipriflavone $(200 \mathrm{mg} / \mathrm{kg} / \mathrm{d})$ once a day for 8 weeks, beginning 4 weeks after ovariectomization. Then, the bone turnover markers, bone biomechanical properties, trabecular architecture, and related protein expressions were evaluated by biochemical assay kits, mechanical testing, microcomputed tomography, immunohistochemical evaluation, and Western blot analysis. Treatment with the flavonoid fraction of Herba Epimedii $(15,30 \mathrm{mg} / \mathrm{kg} / \mathrm{d})$ and ipriflavone $(200 \mathrm{mg} / \mathrm{kg} / \mathrm{d})$ significantly increased bone strength while dramatically inhibiting the serum alkaline phosphatase and tartrate-resistant acid phosphatase levels in ovariectomized rats. Furthermore, the flavonoid fraction of Herba Epimedii also increased osteoprotegerin protein expression and reduced the receptor activator of nuclear factor- $k \mathrm{~B}$ ligand protein expression compared with ovariectomized rats. In addition, the microcomputed tomography results showed that the flavonoid fraction of Herba Epimedii treatment significantly improved trabecular bone mineral density and restored the bone microarchitecture in ovariectomized rats.
Therefore, our results indicated that the flavonoid fraction of Herba Epimedii might be beneficial for improving postmenopausal osteoporosis and should be considered as a promising candidate for treating postmenopausal osteoporosis.

\section{Abbreviations}

$\nabla$

ALP: $\quad$ alkaline phosphatase

BMD: bone mineral density

$\mathrm{BS} / \mathrm{BV}$ : relative bone surface to bone volume

$\mathrm{BV} / \mathrm{TV}$ : relative bone volume to total volume

CT: $\quad$ computed tomography

HEFF: flavonoid fraction of Herba Epimedii

HPLC: high performance liquid chromatogHPLC-ESI-MS: raphy

high performance liquid chromatography-electronic spray ion-mass spectrum

HRT: hormone replacement therapy

IF: ipriflavone

MS: mass spectrum

OPG: osteoprotegerin

OVX: ovariectomized

PBS: $\quad$ phosphate buffered saline

RANKL: receptor activator of nuclear factor $-k B$ ligand

RIPA: radio-immunoprecipitation assay

SERMs: selective estrogen receptor modulators

Tb.N: $\quad$ trabecular number

Tb.Sp: mean trabecular separation

TBST: $\quad$ tris buffered saline with tween-20

Tb.Th: mean trabecular thickness

TRACP: tartrate-resistant acid phosphatase 


\section{Introduction}

\section{$\nabla$}

Osteoporosis is a common skeletal disease with net bone loss, which may be caused by the imbalance of bone resorption and bone formation [1,2]. Osteoporosis is a pressing health issue in the aging population around the world. It has been reported that approximately $40 \%$ of women and $13 \%$ of men aged 50 or older will experience at least one fracture during their remaining lifetime [3]. Traditionally, HRT has been regarded as a gold standard for the prevention and treatment of osteoporosis. However, the epidemiological results from the Women's Health Initiative and the Million Women Study showed that long-term HRT could increase the risk of postmenopausal women to develop stroke, breast cancer, thrombosis, and cardiovascular diseases [4-6]. SERMs are another choice to treat osteoporosis. Unlike estrogens, SERMs have the unique ability to selectively serve as agonists or antagonists in various tissues [7]. Thus, the pharmacological advantage of SERMs is that they can afford the beneficial estrogenic effects in the target tissues and avoid adverse, off-target effects [8]. However, there are still some problems associated with the current SERMs, for example, drug resistance and adverse effects such as hot flashes and blood clots [9]. Therefore, this serves to search for a natural medicine providing an alternative strategy to prevent and treat osteoporosis in postmenopausal women. Herba Epimedii is the dry aboveground part of Epimedium species (Berberidaceae). Epimedium brevicornum Maxim., together with three other Epimedium species, Epimedium sagittatum (Sieb. et Zucc.) Maxim., Epimedium pubescens Maxim., and Epimedium koreaum Nakai, has been recorded in the Chinese Pharmacopoeia [10]. It is widely used as a Chinese medicine for "strengthening the kidney" and "reinforcing bone" for a long time in China and other Asian countries [11,12]. Many studies have demonstrated that the potential anti-osteoporotic activity of Herba Epimedii. Ye et al. found that Herba Epimedii aqueous extracts could prevent bone loss, resulting in a lack of estrogen, by decreasing the expression of tumor necrosis factor- $\alpha$ in monocytes and stimulating the expression of transforming growth factor- $\beta$ [13]. In vitro experiments showed that Herba Epimedii aqueous extracts stimulate cell proliferation and increase ALP activity in rat osteoblast-like UMR 106 cells [14]. Flavonoid is the main bioactive component of Herba Epimedii and attracted many researchers to study its effects and mechanism on osteoporosis. Previous studies by various scientists have repeatedly shown that the total flavone of Epimedium could prevent bone loss and improve BMD in OVX rats [15-17]. Icariin, the major active constituent of Herba Epimedii, is the most commonly used Chinese herbal medicine for the treatment of osteoporosis [18]. Recently, the bonestrengthening activity of icariin has attracted worldwide attention. In vitro studies showed that icariin could stimulate osteoblast differentiation and suppress osteoclast differentiation and bone resorption activity [19-22]. Animal and human studies also confirmed the anabolic effect of icariin in vivo. Wei et al. found that icariin could promote bone formation, increase trabecular numbers, and reduce trabecular separation [23]. The comparing study showed that there was no obvious difference between icariin and estrogen on anti-osteoporotic activity in OVX rats [24]. The main bioactive components of this herb are prenylated flavonol glycosides, and the absorption of flavonol glycosides increased, while the number of glucosides decreased $[25,26]$. Therefore, our laboratory prepared a flavonoid fraction with less glucoside from Herba Epimedii (HEFF) to enhance the effect on the prevention and treatment of osteoporosis. The HEFF included eight com- pounds, epimedoside A, ikarisoside F, baohuoside II, sagittatoside A, sagittatoside B, 7-O-rhamnosyl icariside II, 2"-O-rhamnosyl icariside II, and baohuoside I with a ratio of $3.87 \%, 4.11 \%, 4.19 \%$, $8.14 \%, 32.77 \%, 9.74 \%, 4.11 \%$, and $6.29 \%$, respectively. The eight compounds were composed of about $73.22 \%$ of the flavonoid fraction. The objective of this study was to systematically evaluate the effect of HEFF on OVX rats.

IF is a synthetic isoflavone derivative and commonly been used as an anti-osteoporosis drug in primary osteoporosis patients. The experimental results of many researchers except for Alexandersen et al. suggested that IF is able to prevent bone loss and increase bone mass in postmenopausal women [27-35]. In this study, we used it as a reference drug.

\section{Results \\ $\nabla$}

In order to identify the main compounds of HEFF, an HPLC-ESI-MS assay was performed in this experiment. The HPLC chromatogram of HEFF is shown in 1 Fig. 1 and MS spectrums of the eight compounds in HEFF are shown in 8 Fig. 2. We speculated the possible chemical structures of the eight main compounds according to the chromatographic retention time, relative molecular mass, fragment ion information, and the relevant literature data ( $\odot \mathrm{Ta}-$ ble 1 ). They are epimedoside A, ikarisoside F, baohuoside II, sagittatoside A, sagittatoside B, 7-O-rhamnosyl icariside II, 2"-Orhamnosyl icariside II, and baohuoside I, respectively. Their possible chemical structures are also shown in $\bullet$ Fig. 2.

The biochemical analyses indicated that serum bone formation marker ALP and bone resorption marker TRACP were significantly higher $(+122.61 \%$ and $+110.34 \%$, respectively) in OVX rats compared to the sham group ( $\mathrm{p}<0.01)$. However, administration of HEFF-M (15 mg/kg/d, $30 \mathrm{mg} / \mathrm{kg} / \mathrm{d})$ and IF (200 mg/kg/d) for 8 consecutive weeks attenuated the OVX-induced increase in the levels of ALP and TRACP in a significant manner ( Fig. 3).

A three-point bending test was carried out to evaluate the mechanical properties of the tibia and the results are given in $\bigcirc \mathrm{Ta}$ ble 2. In the OVX group, the tibia showed a lower mechanical strength including ultimate load, modulus of elasticity, and energy to failure ( $p<0.05$ or 0.01 vs. sham). On the other hand, IF (200 mg/kg/d) and HEFF-H (30 mg/kg/d) significantly increased these indices $(\mathrm{p}<0.05)$ after 8 weeks administration. HEFF-M $(15 \mathrm{mg} / \mathrm{kg} / \mathrm{d})$ also markedly enhanced mechanical strength by in-

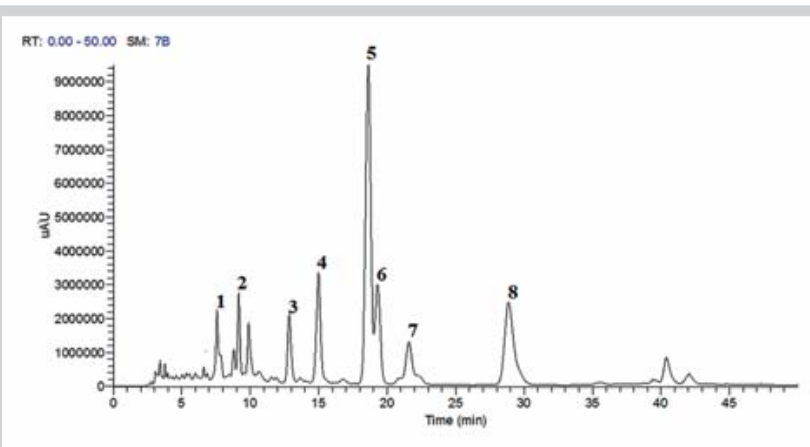

Fig. 1 HPLC chromatograms of HEFF. 1 epimedoside A; 2 ikarisoside F; 3 baohuoside II; 4 sagittatoside A; 5 sagittatoside B; 6 7-O-rhamnosyl icariside II; 7 2" -O-rhamnosyl icariside II; 8 baohuoside I. (Color figure available online only.) 


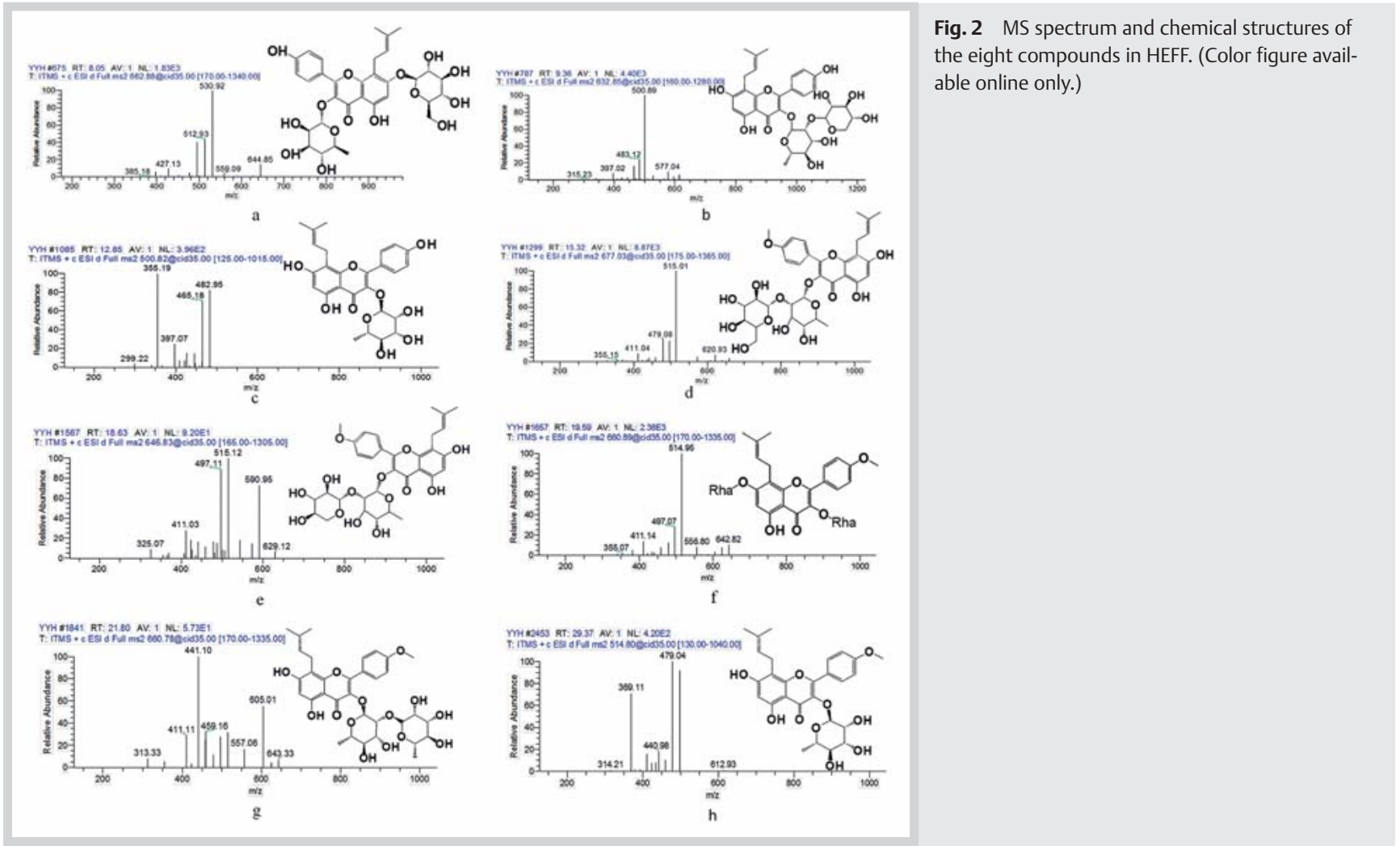

\begin{tabular}{|cclll} 
No. & $\boldsymbol{t}_{\mathrm{R}} / \mathbf{m i n}$ & $\mathbf{( + ) E S I - M S ~} \mathbf{m} / \mathbf{z}$ & Fragment $(\mathbf{m} / \mathbf{z})$ & \multicolumn{1}{c}{ Compound } \\
\hline 1 & 8.05 & 663 & $655,531,513,427$ & epimedoside A \\
\hline 2 & 9.36 & 633 & $577,501,483,397$ & ikarisoside F \\
\hline 3 & 12.95 & 501 & $483,465,397,355$ & baohuoside II \\
\hline 4 & 15.32 & 677 & $631,515,479,411$ & sagittatoside A \\
\hline 5 & 18.63 & 647 & $591,515,497,411$ & sagittatoside B \\
\hline 6 & 19.59 & 661 & $515,497,411$ & 7-O-rhamnosyl icariside II \\
\hline 7 & 21.80 & 661 & $605,557,459,441,411$ & 2 ${ }^{\prime \prime}$-O-rhamnosyl icariside II \\
\hline 8 & 28.37 & 515 & $479,441,369$ & baohuoside I \\
\hline
\end{tabular}

Table 1 The retention time and MS fragmentation ion of the main compounds in HEFF. creasing the modulus of elasticity and energy to failure ( $\mathrm{p}<0.05)$, but had no significant effect on ultimate load.

To further evaluate the effects of HEFF on bone remodeling activity, we analyzed six bone histomorphometric indices including BMD, BV/TV, Tb.N, Tb.Sp, BS/BV, and Tb.Th in all rats by using micro-CT ( Table 3). IF $(200 \mathrm{mg} / \mathrm{kg} / \mathrm{d})$ treatment significantly reversed the effects of ovariectomy on histomorphometric indices by increasing BMD, BV/TV, Tb.N, and Tb.Th, and decreasing Tb. Sp and BS/BV in OVX rats. Although HEFF-L ( $7.5 \mathrm{mg} / \mathrm{kg} / \mathrm{d}$ ) had no significant effect on these six indices, compared with OVX rats, HEFF-H $(30 \mathrm{mg} / \mathrm{kg} / \mathrm{d})$ treatment showed a similar effect to IF $(200 \mathrm{mg} / \mathrm{kg} / \mathrm{d})$ treatment on the six indices, while HEFF-M (15 mg/kg/d) treatment only raised BMD, Tb.N, and Tb.Th and reduced BS/BV. The conclusion mentioned above was further proven by their three-dimensional images of the coronal plane and two-dimensional images of the longitudinal plane obtained from micro-CT ( Fig.4). Compared with the compact and normally distributed trabecular bone in the sham rats, the trabecular bone mass and architecture in the OVX group were significantly deteriorated after 12 weeks, and these indices in the HEFF-M and HEFF-H groups were better than those in the OVX group and similar to those of the IF group.

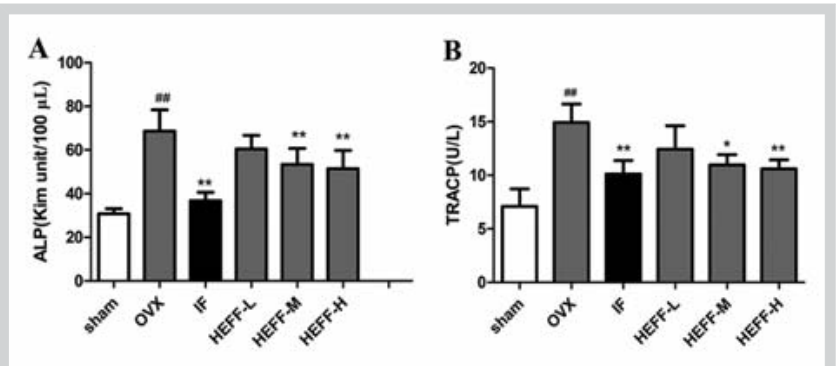

Fig. 3 Effects of HEFF on the bone formation marker $\operatorname{ALP}(\mathbf{A})$ and the bone resorption marker TRACP $(B)$. Data are presented as means $\pm S D(n=8)$. ${ }^{\# \#} \mathrm{P}<0.01$ compared with sham; ${ }^{*} \mathrm{p}<0.05$ compared with $\mathrm{OVX} ;{ }^{* *} \mathrm{p}<0.01$ compared with OVX.

The results of immunophenotypic expressions of OPG and RANKL in the femur of the rats are shown in $\odot$ Fig. 5. OPG and RANKL protein expressions appeared as yellow-brown staining in the cytoplasm. As we can see from 0 Fig. 5 A, B, OPG was significantly reduced, while RANKL was increased markedly in the OVX group 
Table 2 Bone biomechanical parameters.

\begin{tabular}{|lcccccc|}
\hline Parameters & Sham & OVX & IF & HEFF-L & HEFF-M & HEFF-H \\
\hline Ultimate load $(\mathrm{N})$ & $92.75 \pm 5.91$ & $64.25 \pm 8.69^{\#}$ & $85.25 \pm 12.89^{*}$ & $71.25 \pm 12.15$ & $77.75 \pm 6.34$ & $80.75 \pm 7.68^{*}$ \\
\hline Modulus of elasticity (MPa) & $176.68 \pm 18.97$ & $110.09 \pm 17.02^{\# \#}$ & $162.88 \pm 13.40^{*}$ & $127.37 \pm 25.96$ & $145.97 \pm 17.21^{*}$ & $154.27 \pm 16.21^{*}$ \\
\hline Energy to failure (mJ) & $27.50 \pm 3.83$ & $12.56 \pm 2.98^{\# \#}$ & $22.68 \pm 1.81^{* *}$ & $14.70 \pm 2.62$ & $18.30 \pm 1.99^{*}$ & $20.73 \pm 3.66^{*}$ \\
\hline
\end{tabular}

The data are expressed as the means \pm SD; ${ }^{\#} p<0.05,{ }^{\# \#} \mathrm{p}<0.01$ compared with sham; ${ }^{*} \mathrm{p}<0.05,{ }^{* *} \mathrm{p}<0.01$ compared with $\mathrm{OVX}$

Table 3 Micro-CT analysis of BMD, BV/TV, Tb.N, Tb.Sp, BS/BV, and Tb.Th.

\begin{tabular}{|c|c|c|c|c|c|c|}
\hline Parameters & Sham & OVX & IF & HEFF-L & HEFF-M & HEFF-H \\
\hline $\operatorname{BMD}\left(\mathrm{mg} / \mathrm{cm}^{3}\right)$ & $442.37 \pm 18.13$ & $160.89 \pm 18.12^{\# \#}$ & $312.38 \pm 26.10^{* *}$ & $178.38 \pm 17.4$ & $249.17 \pm 33.37^{*}$ & $261.19 \pm 44.89^{*}$ \\
\hline BV/TV (\%) & $18.20 \pm 0.53$ & $11.85 \pm 1.73^{\# \#}$ & $15.97 \pm 2.01^{*}$ & $12.17 \pm 3.82$ & $14.10 \pm 1.34$ & $15.01 \pm 1.67^{*}$ \\
\hline Tb.N (1/mm) & $1.26 \pm 0.33$ & $0.48 \pm 0.11^{\# \#}$ & $0.83 \pm 0.04^{* *}$ & $0.50 \pm 0.09$ & $0.63 \pm 0.70^{*}$ & $0.71 \pm 0.05^{*}$ \\
\hline Tb.Sp (mm) & $0.68 \pm 0.13$ & $1.91 \pm 0.42^{\# \#}$ & $1.09 \pm 0.13^{* *}$ & $1.71 \pm 0.18$ & $1.48 \pm 0.35$ & $1.16 \pm 0.17^{*}$ \\
\hline $\mathrm{BS} / \mathrm{BV}(1 / \mathrm{mm})$ & $8.04 \pm 0.69$ & $14.95 \pm 1.33^{\# \#}$ & $10.33 \pm 1.16^{* *}$ & $13.25 \pm 1.07$ & $11.13 \pm 0.88^{* *}$ & $10.71 \pm 0.75^{* *}$ \\
\hline Tb.Th $(\mu \mathrm{m})$ & $250.28 \pm 21.45$ & $150.71 \pm 39.70^{\# \#}$ & $236.01 \pm 33.11^{*}$ & $176.01 \pm 24.56$ & $200.69 \pm 15.76^{*}$ & $225.51 \pm 28.11^{*}$ \\
\hline
\end{tabular}

The data are expressed as the means $\pm \mathrm{SD} ;{ }^{\#} \mathrm{p}<0.05,{ }^{\# \#} \mathrm{p}<0.01$ compared with sham; ${ }^{*} \mathrm{p}<0.05,{ }^{* *} \mathrm{p}<0.01$ compared with OVX

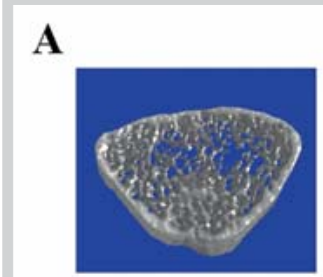

$\mathbf{a}$

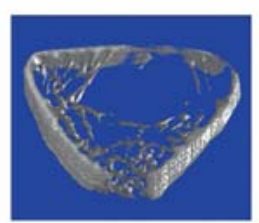

d

B

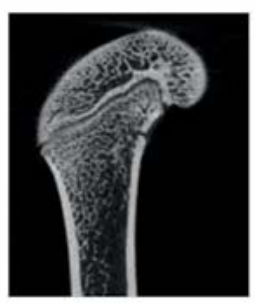

a

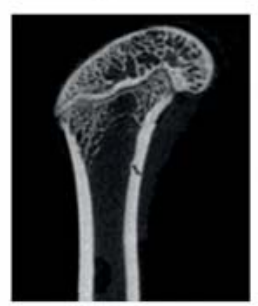

d

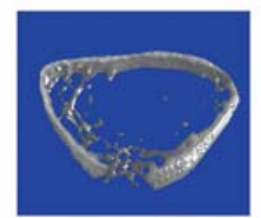

b

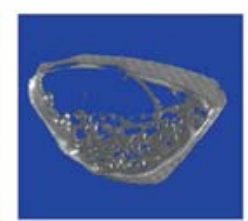

e

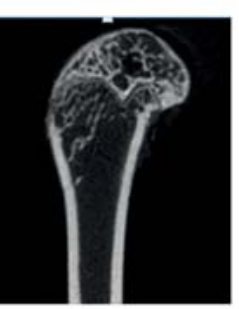

b

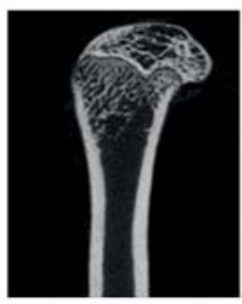

e

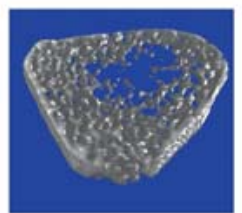

c

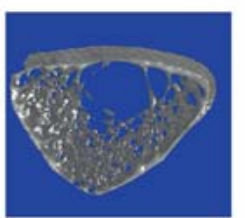

f

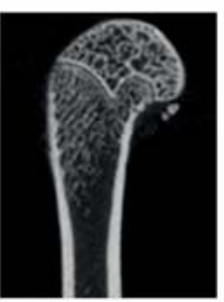

$\mathrm{c}$

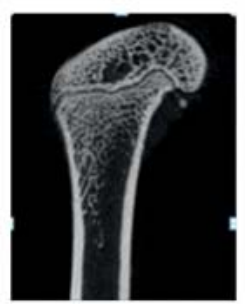

f
Fig. 4 Representative three-dimensional (A) or two-dimensional (B) micro-CT images of trabecular bone from the distal femur with the sham and different OVX treatments; a sham group; b OVX group; c IF group; d HEFF-L (7.5 mg/kg/d); e HEFF-M (15 mg/kg/d); f HEFF-H (30 mg/kg/d). (Color figure available online only.)

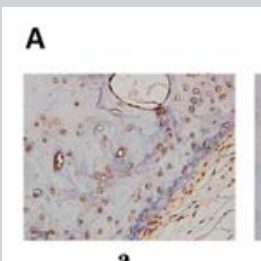

a

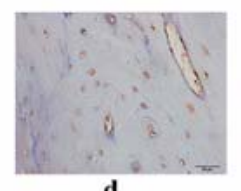

d

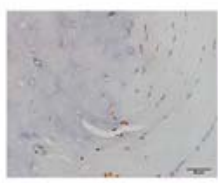

b
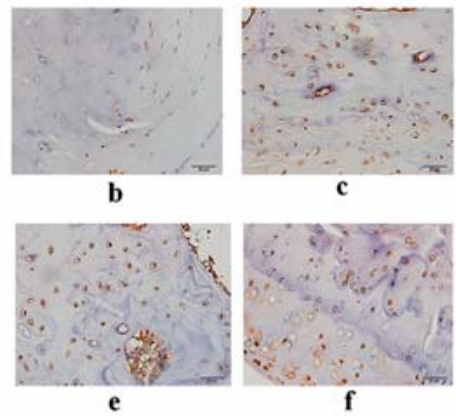

B
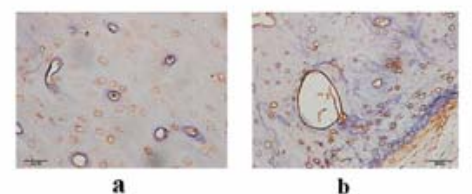

b
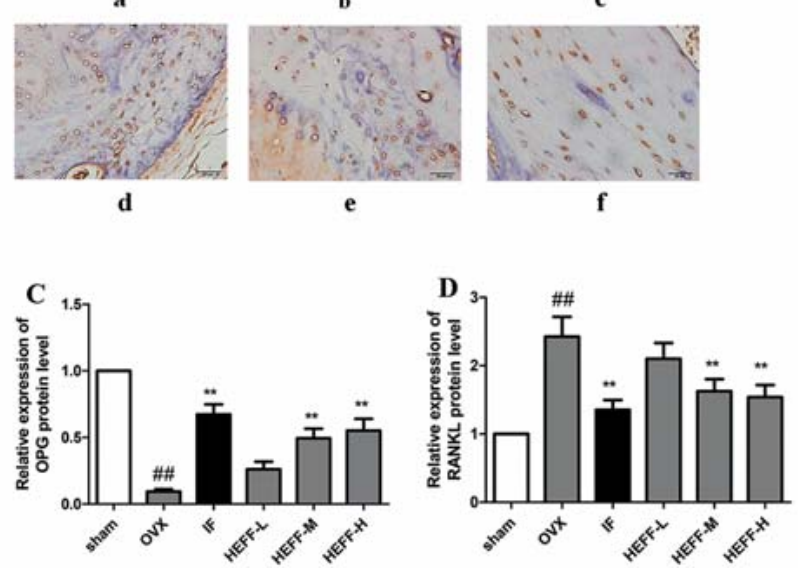

Fig. 5 Representative immunohistochemical staining of the femora from osteoporotic rats. A OPG protein expression. B RANKL protein expression; a sham group; b OVX group; c IF group; d HEFF-L $(7.5 \mathrm{mg} / \mathrm{kg} / \mathrm{d})$; e HEFF-M $(15 \mathrm{mg} / \mathrm{kg} / \mathrm{d}) ; \mathbf{f}$ HEFF-H (30 mg/kg/d). C Relative expression of OPG protein level. D Relative expression of RANKL protein levels. (Color figure available online only.) 


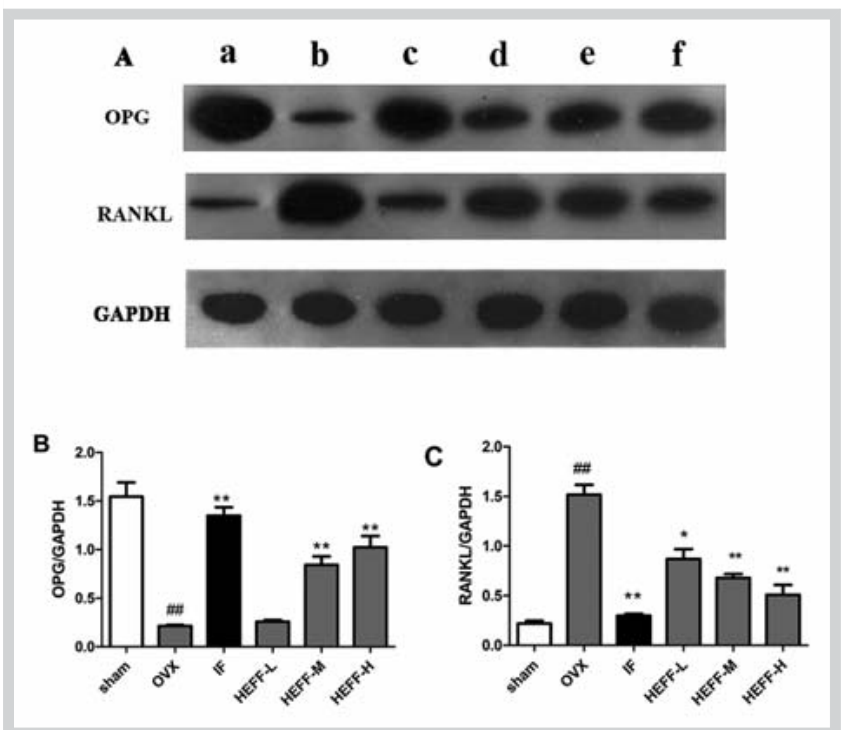

Fig. 6 Western blot analysis of the femora from osteoporotic rats. A a sham group; b OVX group; c IF group; d HEFF-L (7.5 mg/kg/d); e HEFF-M $(15 \mathrm{mg} / \mathrm{kg} / \mathrm{d})$; $\mathbf{f}$ HEFF-H $(30 \mathrm{mg} / \mathrm{kg} / \mathrm{d})$. B OPG protein level. C RANKL protein level. Data are expressed as the means \pm SD, $n=3$. ${ }^{\#} \mathrm{P}<0.01$ compared with sham; ${ }^{*} \mathrm{p}<0.05$ compared with OVX; ${ }^{* *} \mathrm{p}<0.01$ compared with OVX.

compared with the sham group. However, this decrease or increase can be blocked by treatment with IF $(200 \mathrm{mg} / \mathrm{kg} / \mathrm{d})$ or HEFF (15 mg/kg/d and $30 \mathrm{mg} / \mathrm{kg} / \mathrm{d}$ ) for 8 weeks. Quantitative results shown in $\odot$ Fig. 5C, D also confirmed the descriptive observations.

Western blot analysis using antibodies against OPG and RANKL was used to measure the femur levels of these proteins. As shown in $\odot$ Fig. 6 A, B, OPG protein expression was dramatically reduced in the OVX group ( $p<0.01$ vs. sham). However, this reduction was significantly increased by treatment with IF $(200 \mathrm{mg} / \mathrm{kg} / \mathrm{d})$ and HEFF $(15 \mathrm{mg} / \mathrm{kg} / \mathrm{d}, 30 \mathrm{mg} / \mathrm{kg} / \mathrm{d}$ ) when compared with OVX $(p<0.01)$. Thus, these results demonstrated that HEFF plays an effective role in OPG protein expression that was reduced by ovariectomy. In contrast, an obvious increase of RANKL protein was observed in the OVX group compared to the sham group $(\mathrm{p}<0.01)$. Interestingly, IF (200 mg/kg/d) and HEFF $(7.5 \mathrm{mg} / \mathrm{kg} / \mathrm{d}, 15 \mathrm{mg} / \mathrm{kg} /$ d, $30 \mathrm{mg} / \mathrm{kg} / \mathrm{d}$ ) could significantly downregulate the overexpression of RANKL protein in a dose-dependent manner ( $p<0.05$ or $\mathrm{p}<0.01$ vs. OVX) ( $\odot$ Fig. $6 \mathrm{~A}, \mathrm{C}$ ). These results suggested that HEFF could cause a downregulation of RANKL protein expression induced by ovariectomy.

\section{Discussion}

$\nabla$

Osteoporosis has become a serious challenge to health and quality of life of the aged in view of low bone mineral density and increased fracture risk. The therapeutic approaches for the prevention and treatment of osteoporosis has been used in clinical practice with the availability of effectiveness, such as HRT, which has been confirmed to be associated with a high risk of incidence of dramatic side effects. Alternative medicine has attracted the attention of many researchers because of its efficacy and safety in patients with osteoporosis. Herba Epimedii, a traditional Chinese medicine, has been widely used as an anti-osteoporosis herb for the prevention and treatment of osteoporotic fractures [36]. In the present study, the potential anti-osteoporosis activity of HEFF has been evaluated in vivo in OVX rats. The study provided important information on the anti-osteoporosis activity of HEFF.

As we all know, prenylated flavonoids, which include triglycosides, diglycosides, and monoglycoside, are the main active constituents of Herba Epimedii. Some previous researches used a Caco-2 model and the rat intestinal perfusion model to study the absorption and metabolism of prenylated flavonoids from Herba Epimedii in the rat intestine [26]. The results indicated that triglycosides and diglycosides might be hydrolyzed as secondary glycosides in the intestine before absorption, while monoglycoside (baohuoside I) could be absorbed by enterocytes directly. They also found that the absorption of monoglycoside is better than diglycoside (e.g., icariin), and diglycoside is better than triglycoside (e.g., epimedin A, epimedin B, and epimedin C) [25]. Our previous study confirmed that cellulose could hydrolyze triglycosides and diglycosides could translate into secondary glycosides $[37,38]$. In this study, we used the crude extract (50\% ethanolic) of Herba Epimedii as the raw material to prepare HEFF through enzymolysis, separation, and purification. Compared with the crude extract of Herba Epimedii, all of the triglycosides disappeared, while the diglycosides and monoglycoside increased sharply in HEFF. Therefore, we assumed that HEFF, which included eight diglycosides or monloglycoside, could be easily absorbed in intestines. Subsequently, we validated its anti-osteoporosis activity by using an OVX model.

Estrogen deficiencies are the important risk factors in the pathogenesis of osteoporosis. Therefore, OVX rats have been widely used as a model for postmenopausal osteoporosis [39,40]. IF, a derivative of phytoestrogens, was included in the study as a reference compound for the effect of bone modeling and remodeling. Because of the ovarian hormone deficiency, ovariectomy resulted in a dramatic decrease in biomechanical strength and bone quality, consistent with a previous study [41]. HEFF treatments at the doses of 15 and $30 \mathrm{mg} / \mathrm{kg} / \mathrm{d}$ could significantly increase the OPG protein level, biomechanical strength, and bone quality as well as decrease serum ALP, TRACP, and RANKL protein levels. These results suggested that HEFF had a similar effect as IF on serum ALP, serum TRACP, OPG, RANKL protein level, biomechanical strength, and bone quality. It indicated that HEFF possessed anti-osteoporotic activity in osteoporotic rats induced by estrogen deficiency.

The serum activity of ALP and TRACP are useful markers of bone formation and bone resorption, respectively. Other investigators have indicated that the levels of ALP and TRACP were increased in the OVX group compared with the sham group because of estrogen deficiency $[42,43]$. A similar change was observed in this study. However, treatment with HEFF at higher doses (15 or $30 \mathrm{mg} / \mathrm{kg} / \mathrm{d}$ ) significantly reversed the changes of ALP and TRACP. The results showed that HEFF inhibited bone loss induced by ovariectomy, probably by stimulating bone formation and inhibiting bone resorption.

Bone remodeling demands a precise balance between bone resorption and formation. The RANKL/RANK/OPG system plays an important role in regulating the balance between the activity of osteoblasts and osteoclasts [44]. RANKL binds to its cellular receptor RANK to promote the differentiation of preosteoclasts into mature osteoclasts and increase bone resorption, while OPG serves as a decoy receptor of RANKL and inhibits its activation of osteoclastogenesis. In this study, we found that the levels of OPG protein were increased, while the levels of RANKL protein were decreased by treatment with HEFF. The results indicated that 
one of the mechanisms of HEFF inhibiting bone loss was associated with an increased OPG/RANKL ratio.

The most important marker of osteoporosis is the severe decrease in bone strength, which directly results in bone fractures [45]. The three-point bending test has been commonly used to evaluate bone strength [46], which has shown that bending ultimate load, modulus of elasticity, energy to failure, and stiffness are good indicators of the mechanical load of cortical bone [47]. In this study, the results of the three-point bending test of tibial diaphysis showed that the administration of HEFF at a higher dosage to OVX animals produced a significant increase in bone strength.

BMD is the golden standard to diagnose osteoporosis. In the present study, we used Micro-CT to measure BMD and evaluate bone histomorphometry $[48,49]$. The micro-CT analysis of the distal femur in OVX rats reflected changes in the bone quality through higher porosity, trabecular separation, lower BMD, trabecular thickness, trabecular connectivity, and trabecular number [50]. Interestingly, treatment with IF or HEFF at higher doses for 8 weeks was able to partly reversed the changes induced by OVX (๑ Table 3). This result further explains the change of bone strength evaluated by the biomechanical test.

In conclusion, this study demonstrated for the first time that HEFF, a flavonoid fraction of Herba Epimedii, had powerful protective effects on OVX-induced osteoporosis in rats by stimulating bone formation and inhibiting bone turnover and bone resorption. Our experimental results suggest that HEFF supplementation could be an alternative to HRT for the prevention of postmenopausal osteoporosis.

\section{Material and Methods}

V

\section{Plant material}

From Herba Epimedii, the dried leaves of E. koreaum (Batch No. 20140206) were authenticated by Professor B.L. Guo from the Institute of Medicinal Plant Development, Chinese Academy of Medical Sciences. A voucher specimen (No. HE-20140206) was deposited at Jiangsu Provincial Academy of Chinese Medicine. IF tablets (Batch No.140201) containing $46.42 \%$ IF were purchased from a pharmacy.

\section{Preparation of the flavonoid fraction of Herba Epimedii} Herba Epimedii $(5 \mathrm{~kg}$ ) was refluxed with $90 \mathrm{~L} 50 \%$ ethanol and $75 \mathrm{~L}$ $50 \%$ ethanol $(\mathrm{v} / \mathrm{v})$ for $1 \mathrm{~h}$, respectively. All of the extracts were combined together and ethanol was removed under reduced pressure, then enzymolysis by cellulose was carried out (cellulose: total flavonoids $=1: 7$ ). The enzymatic hydrolysates were precipitated by adding ethanol to a ratio of ethanol: water $=60: 40(\mathrm{v} / \mathrm{v})$ and then they were filtered. Next, the supernatant of this extract was concentrated and loaded onto the macroporous resin AB-8 column. After adsorption overnight, the adsorbate-laden column was eluted with different concentrations of ethanol $(0 \%, 30 \%$, $60 \%, 80 \%$ ) at a flow rate of $2 \mathrm{BV} / \mathrm{h}$. Then, $60 \%$ and $80 \%$ ethanol eluent were combined and concentrated, stewing overnight. The deposit was redissolved in $95 \%$ ethanol and centrifuged at $4000 \mathrm{rpm}$ for $10 \mathrm{~min}$, while the supernatant was taken to be concentrated and dried at $50^{\circ} \mathrm{C}$, and then the HEFFs were obtained.

\section{Chromatographic conditions and equipment}

HPLC analysis of the samples was performed on an Agilent 1200 series system, equipped with an Agilent SB- $\mathrm{C}_{18}$ column $(250 \mathrm{~mm}$ $\times 4.6 \mathrm{~mm}, 5 \mu \mathrm{m}$ ). Acetonitrile (A) and $0.1 \%$ formic acid (B) were used for the mobile phase. The gradient program was set as follows: 0-30 min, 35\% A, 30-35 min, 35-40\% A, 35-50 min, 40\% A. The flow rate was $1.0 \mathrm{~mL} / \mathrm{min}$, while the column temperature was kept at $30^{\circ} \mathrm{C}$. The sample was detected at $270 \mathrm{~nm}$ with an injection volume of $10 \mu \mathrm{L}$.

HPLC-ESI-MS analysis was operated in the positive mode. The optimized operating parameters were as follows: ion spray voltage: $4.5 \mathrm{kV}$; heated capillary temperature: $300^{\circ} \mathrm{C}$; capillary voltage: $5 \mathrm{~V}$; auxiliary gas $\left(\mathrm{N}_{2}\right)$ pressure: 10 arbitrary units; sheath gas $\left(\mathrm{N}_{2}\right)$ pressure: 40 arbitrary units. The mass spectrometer was detected over a range of $m / z 80$ to 2000 in the full scan mode.

\section{Animals}

All procedures involving animals were approved by the Animal Experimentation Ethics Committee of Jiangsu Provincial Academy of Chinese Medicine (approval No. SYXK (su) 2011-0017, Nov. 28, 2011). Forty-eight 3-month-old female Sprague Dawley rats were purchased from the animal center of Nantong University. The rats were housed four per cage in an air-conditioned room at $25 \pm 1{ }^{\circ} \mathrm{C}, 55-60 \%$ relative humidity, and $12 \mathrm{~h}$ of light alternating with $12 \mathrm{~h}$ of darkness (lights on from $08: 00 \mathrm{~h}$ to 20:00 h) with ad libitum access to food and water.

\section{Experimental groups}

One week after arrival, the rats underwent either bilateral laparotomy (sham, $\mathrm{n}=8$ ) or bilateral OVX (OVX, $\mathrm{n}=40$ ). Four weeks after ovariectomization, OVX rats were randomly divided into five groups as follows: OVX treated with vehicle $(n=8, O V X)$, OVX treated with IF ( $\mathrm{n}=8, \mathrm{IF}, 200 \mathrm{mg} / \mathrm{kg} / \mathrm{d})$, and OVX treated with a low $(\mathrm{n}=8$, HEFF-L, $7.5 \mathrm{mg} / \mathrm{kg} / \mathrm{d})$, medium $(\mathrm{n}=8$, HEFF-M, $15 \mathrm{mg} / \mathrm{kg} / \mathrm{d}$ ), or high $(\mathrm{n}=8, \mathrm{HEFF}-\mathrm{H}, 30 \mathrm{mg} / \mathrm{kg} / \mathrm{d}$ ) dose of HEFF. HEFF and IF were suspended in $0.5 \%$ sodium carboxymethyl cellulose and administrated by oral gavage, which started from the 5th week (29th day) after OVX to the 8th week (84th day). At the end of the 8-week study period, blood was collected from the orbit and allowed to clot, then the blood samples were centrifuged for serum isolation and then stored at $-80^{\circ} \mathrm{C}$ for biochemical analysis. The left femurs were put in $4 \%$ neutral formaldehyde solution for micro-CT scanning and immunohistochemical evaluation. The right femora were stored at $-80^{\circ} \mathrm{C}$ for Western blot analysis. The left tibias were stored at $-20^{\circ} \mathrm{C}$ and subjected to mechanical testing.

\section{Bone turnover markers evaluation}

The serum bone formation marker (ALP) and bone resorption marker (TRACP) were analyzed by biochemical assays. Serum samples were centrifuged at $3000 \mathrm{rpm}$ for $10 \mathrm{~min}$ at $37^{\circ} \mathrm{C}$, and the serums were stored at $-80^{\circ} \mathrm{C}$ until use. Next, these markers were assayed using a commercial kit according to the manufacturer's instructions.

\section{Biomechanical evaluation}

The right tibias were harvested, cleaned of superficial tissues, incubated in PBS, and frozen at $-20^{\circ} \mathrm{C}$ until assessed for the threepoint bending test using an Instron 5943 material testing system [51]. The two supports were separated by a distance of $12.1 \mathrm{~mm}$ and a central bending load was applied at a displacement rate of $1 \mathrm{~mm} / \mathrm{min}$ until the tibia was broken [52]. The force-displace- 
ment curve of each test was acquired by the computer, which was connected to the apparatus. Force and deformation data were collected and analyzed to calculate the ultimate load (N), modulus of elasticity (MPa), and energy to failure (mJ).

\section{Trabecular bone microarchitecture measurement by microcomputer tomography}

The left femora were fixed in $4 \%$ paraformaldehyde for $48 \mathrm{~h}$ and scanned using a cone beam (Skyscan1176, Skyscan). The X-ray generator was set at a voltage of $50 \mathrm{KV}$ with a current of $500 \mu \mathrm{A}$, which produced images with an isotropic spatial resolution of $\left(35 \mathrm{~mm}^{3}\right)$ voxel size. For analysis of the trabecular bone, 50 consecutive slices $(1.75 \mathrm{~mm})$ were selected as the region of interest beginning $6.44 \mathrm{~mm}$ away from the distal femur growth plate. The following parameters were measured using a three-dimensional region of interest by Skyscan analysis software: BMD, BV/ TV, Tb.N, Tb.Sp, BS/BV, and Tb.Th.

\section{Immunohistochemical staining}

After being scanned by micro-CT, immunohistochemical assessment was conducted to determine the protein expressions of OPG and RANKL according to the following procedure. The left femora were embedded in paraffin and cut into 5- $\mu \mathrm{m}$ thick sections, deparaffinized, and rehydrated. After being washed with PBS three times, the slices were incubated with $0.3 \%$ hydrogen peroxide for $15 \mathrm{~min}$ to quench endogenous peroxidase. Next, the samples were incubated with primary antibodies (dilution $1: 200)$ overnight at $4{ }^{\circ} \mathrm{C}$. After being washed three times with PBS, slices were incubated with the secondary antibody for 30 min followed by 3,3'-diaminobenzidine development. Fifteen random images from three sections per animals were obtained using a BX51 Olympus microscope equipped with a DP71 camera. Each image was captured under the same conditions. Quantitative analysis was determined using image analysis software Image pro-Plus 6.0. The fields were selected and analyzed by two blinded examiners. When there was a difference, a consensus was reached by discussion.

\section{Western blot analysis}

The right femora were lysed in RIPA lysis buffer containing a protease inhibitor cocktail. The samples were sonicated and incubated on ice for $30 \mathrm{~min}$ and then the insoluble material was removed by centrifuging at $12000 \mathrm{rpm}$ for $5 \mathrm{~min}$. The protein in the supernatant was collected for Western blot analysis. Protein concentrations of each sample were measured using the BCA protein assay kit. Equal quantities of protein were separated on a $10 \%$ polyacrylamide-sodium dodecyl sulfate gel and transferred to polyvinylidene fluoride membranes. Nonspecific binding sites were blocked in TBST containing 5\% nonfat milk and 0.1\% Tween 20 for $2 \mathrm{~h}$ at room temperature. After being washed three times with TBST, the membranes were incubated with primary antibodies raised against RANKL $(1: 1000)$, OPG $(1: 1000)$, and GAPDH $(1: 1000)$ at $4{ }^{\circ} \mathrm{C}$ overnight. Membranes were washed with TBST three times and then incubated with secondary antibodies $(1: 5000)$ conjugated to horseradish peroxidase. Immunoreactive proteins were detected by enhanced chemiluminescence reagent. The Western blot experiments were repeated three times using different samples. The densities of the bands were determined using image analysis software Gel-pro Analyzer 4.5 .

\section{Statistical evaluation}

Data are expressed as the means \pm standard deviation (SD). Oneway analysis of variance (ANOVA) in IBM SPSS 11.5 software was used for measuring statistically significant differences between groups for all outcomes. Values of $\mathrm{p}<0.05$ were considered statistically significant.

\section{Acknowledgements}

$\nabla$

We gratefully acknowledge the financial support from the Suzhou Science and Technology program (ZXY2013022), the National Natural Science Foundation of China (No.81274088 and No. 81303275 ) and the Special Public Welfare Industry of Traditional Chinese Medicine Research (No. $201507004-10$ )

\section{Conflict of Interest}

$\nabla$

The authors declare no conflict of interest.

\section{References}

1 Sambrook P, Cooper C. Osteoporosis. Lancet 2006; 367: 2010-2018

2 Khosla S, WestendorfJJ, Oursler MJ. Building bone to reverse osteoporosis and repair fractures. J Clin Invest 2008; 118: 421-428

3 Li XF, Xu H, Zhao YJ, Tang DZ, Xu GH, Holz J, Wang J, Cheng SD, Shi Q Wang YJ. Icariin augments bone formation and reverses the phenotypes of osteoprotegerin-deficient mice through the activation of Wnt/ $\beta$-catenin-BMP signaling. Evid Based Complement Alternat Med 2013; 2013: 652317

4 Rossouw JE, Anderson GL, Prentice RL, LaCroix AZ, Kooperberg C, Stefanick ML, Jackson RD, Beresford SA, Howard BV, Johnson KC, Kotchen JM, Ockene J. Writing group for the Women's Health Initiative investigators. Risks and benefits of estrogen plus progestin in healthy postmenopausal women: principal results from the Women's Health Initiative randomized controlled trial. JAMA 2002; 288: 321-333

5 Beral V; Million Women Study Collaborators. Breast cancer and hormone-replacement therapy in the Million Women Study. Lancet 2003; 362: 419-427

6 Gambacciani M, Ciaponi M, Genazzani AR. The HRT misuse and osteoporosis epidemic: a possible future scenario. Climacteric 2007; 10: 273-275

$7 \mathrm{Komm} \mathrm{BS}$. A new approach to menopausal therapy: the tissue selective estrogen complex. Reprod Sci 2008; 15: 984-992

8 Stephen M, Darshan S, Sonia LP, John AA. Selective estrogen receptor modulators: tissue specificity and clinical utility. Clin Interv Aging 2014; 9: 1437-1452

9 Jordan VC. Selective estrogen receptor modulation: concept and consequences in cancer. Cancer Cell 2004; 5: 207-213

10 Pharmacopoeia Commission of PRC. Pharmacopoeia of the People's Republic of China. Beijing: Chinese Medical Science and Technology Press; 2010: 306

11 An SJ, Li T, Li E. Effect of kidney-tonifying herbs on ovary function and bone mass in postmenopausal women. Chin J Osteoporosis 2002; 6: 55-59

12 Wang JS, Xu X, Jin JS, Chen GJ, Cao HY, Liu JF, Mao KC, Wang GC. Clinical study of treatment of female osteoporosis with Yishen Jiangu pills. Chin J Osteoporosis 1997; 3: 61-63

$13 \mathrm{Ye}$ C, Su J, Wang F. Expression of TNF- $\alpha$ and TGF- $\beta_{1}$ in vertebrae of model rats of epimedium preventing ovariectomy-induced osteoporosis. Chin J Clin Anat 2006; 24: 687-690

14 Xie F, Wu CF, Lai WP, Yang XJ, Cheung PY, Yao XS, Leung PC, Wong MS. The osteoprotective effect of Herba epimedii (HEP) extract in vivo and in vitro. Evid Based Complement Alternat Med 2005; 2: 353-361

15 Chen BL, Xie DH, Wang ZW, Li FB, Xu DL, Li YQ. Effect of total flavone of Epimedium on expression of bone OPG, OPGL $\mathrm{mRNA}$ in ovariectomized rats. Zhongguo Gu Shang 2009; 22: 271-273

16 Zhang G, Qin L, Shi Y. Epimedium-derived phytoestrogen flavonoids exert beneficial effect on preventing bone loss in late postmenopausal 
women: a 24-month randomized, double-blind and placebo-controlled trial. J Bone Miner Res 2007; 22: 1072-1079

17 Jiang YN, Mo HY, Chen JM. Effects of Epimedium total flavonoids phytosomes on preventing and treating bone loss of ovariectomized rats. Zhongguo Zhong Yao Za Zhi 2002; 27: 221-224

18 Kapoor S. Icariin and its emerging role in the treatment of osteoporosis. Chin Med J (Engl) 2013; 126: 400

19 Ma HP, Ming LG, Ge BF, Zhai YK, Song P, Xian CJ, Chen KM. Icariin is more potent than genistein in promoting osteoblast differentiation and mineralization in vitro. J Cell Biochem 2011; 112: 916-923

20 Zhang D, Zhang J, Fong C, Yao X, Yang M. Herba Epimedii flavonoids suppress osteoclastic differentiation and bone resorption by inducing $\mathrm{G} 2 /$ M arrest and apoptosis. Biochimie 2012; 94: 2514-2522

21 Huang J, Yuan L, Wang X, Zhang TL, Wang K. Icaritin and its glycosides enhance osteoblastic, but suppress osteoclastic, differentiation and activity in vitro. Life Sci 2007; 81: 832-840

22 Song L, Zhao J, Zhang X, Li H, Zhou Y. Icariin induces osteoblast proliferation, differentiation and mineralization through estrogen receptormediated ERK and JNK signal activation. Eur J Pharmacol 2013; 714: $15-22$

23 Wei H, Zili L, Yuanlu C, Biao Y, Cheng L, Xiaoxia W, Yang L, Xing W. Effect of icariin on bone formation during distraction osteogenesis in the rabbit mandible. Int J Oral Maxillofac Surg 2011; 40: 413-418

24 Yang $L, Y u Z$, Qu H, Li M. Comparative effects of hispidulin, genistein, and icariin with estrogen on bone tissue in ovariectomized rats. Cell Biochem Biophys 2014; 70: 485-490

25 Chen Y, Jia XB, Hu M. Absorption and transportation of flavonoids in Herb Epimedii across Caco-2 monolayer model. Chin Tradit Herb Drugs 2009; 40: 220-224

26 Chen Y, WangJY, Jia XB, Tan XB, Hu M. Role of intestinal hydrolase in the absorption of prenylated flavonoids present in Yin Yanghuo. Molecules 2011; 16: 1336-1348

27 Alexandersen P, Toussaint A, Christiansen C, Devogelaer JP, Roux C, Fechtenbaum J, Gennari C, Reginster JY. Ipriflavone in the treatment of postmenopausal osteoporosis. JAMA 2001; 285: 1482-1488

28 Gennari C, Agnusdei D, Crepaldi G, Isaia G, Mazzuoli G, Ortolani S, Bufalino L, Passeri M. Effect of ipriflavone - a synthetic derivative of natural isoflavones - on bone mass loss in the early years after menopause. Menopause 1998; 5: 9-15

29 Adami S, Buffalino L, Cervetti R, Di Marco C, Di Munno O, Fantasia L, Isaia GC, Serni U, Vecchiet L, Passeri M. Ipriflavone prevents radial bone loss in postmenopausal with low bone mass over 2 years. Osteoporos Int 1997; $7: 119-125$

30 Gennari C, Adami S, Agnusdei D, Bufalíno L, Cervetti R, Crepaldi G, Di Marco C, Di Munno O, Fantasia L, Isaia GC, Mazzuoli GF, Ortolani S, Passeri $M$, Serni $U$, Vecchiet $L$. Effect of chronic treatment with ipriflavone in postmenopausal women with low bone mass. Calcif Tissue Int 1997; 61: S19-S22

31 Ohta H, Komukai S, Makita K, Masuzawa T, Nozawa S. Effects of 1-year ipriflavone treatment on lumbar bone mineral density and bone metabolic markers in postmenopausal women with low bone mass. Horm Res 1999: 51: 178-183

32 Agnusdei D, Zacchei F, Bigazzi S, Cepollaro C, Nardi P, Montagnani M, Gennari C. Metabolic and clinical effects of ipriflavone inestablished post-menopausal osteoporosis. Drugs Exp Clin Res 1989; 15: 97-104

33 Agnusdei D, Bufalino L. Efficacy of ipriflavone in established osteoporosis and long-term safety. Calcif Tissue Int 1997; 61: 23-27

34 Agnusdei D, Crepaldi G, Isaia G, Mazzuoli G, Ortolani S, Passeri M, Bufalino L, Gennari C. A double blind, placebo-controlled trial of ipriflavone for prevention of postmenopausal spinal bone loss. Calcif Tissue Int 1997; 61: 142-147
35 Passeri M, Biondi M, Costi D, Bufalino L, Castiglione GN, Di Peppe C, Abate $G$. Effect of ipriflavone on bone mass in elderly osteoporotic women. Bone Miner 1992; 19: S57-S62

36 Zhai YK, Guo X, Pan YL, Niu YB, Li CR, Wu XL, Mel QB. A systematic review of the efficacy and pharmacological profile of Herba Epimedii in osteoporosis therapy. Pharmazie 2013; 68: 713-722

37 Jia DS, Jia XB, Zhao JL, Shi F, Jiang J, Huang Y. Preparation of baohuoside I by enzymolysis of icariin with cellulose. Chin Tradit Herb Drugs 2010; 41: 888-892

38 Xu FJ, Sun E, Zhang ZH, Cui L, Jia XB. The research of preparation of sagittatoside B by enzymolysis of epimedin B with cellulose. Chin J Chin Mater Med 2014; 39: 235-238

39 Jiang J, Li J, Jia XB. The antiosteoporotic activity of central-icaritin (CIT) on bone metabolism of ovariectomized rats. Molecules 2014; 19: 18690-18704

40 Lelovas PP, Xanthos TT, Thoma SE, Lyritis GP, Dontas IA. The laboratory rat as an animal model for osteoporosis research. Comp Med 2008; 58: 424-430

41 Shih CC, Wu YW, Lin WC. Ameliorative effects of Anoectochilus formosanus extract on osteopenia in ovariectomized rats. J Ethnopharmacol 2001; 77: 233-238

42 Bohatyrewicz A. The effect of sodium fluoride on selected biochemicalmarkers of bone turnover in ovariectomized rats. Pol Merkur Lekarski 1998; 5: 192-194

43 Zhang DW, Deng HL, Qi W, Zhao GY, Cao XR. Osteoprotective effect of cordycepin on estrogen deficiency-induced osteoporosis in vitro and in vivo. Biomed Res Int, advance online publication 22 March 2015; DOI: $10.1155 / 2015 / 423869$

44 Boyce BF, Xing L. Functions of RANKL/RANK/OPG in bone modeling and remodeling. Arch Biochem Biophys 2008; 473: 139-146

45 Liu XL, Lei W, Wu ZX, Cui Y, Han BJ, Fu SC, Jiang CL. Effects of glucocorticoid on BMD, micro-architecture and biomechanics of cancellous and cortical bone mass in OVX rabbits. Med Eng Phys 2012; 34: 2-8

46 Fridoni M, Nejati H, Salimi M, Gharavi SM, Bayat M, Amini A, Torkman G, Bayat S. Evaluation of the effects of LLLT on biomechanical properties of tibial diaphysis in two rat models of experimental osteoporosis by a three point bending test. Lasers Med Sci 2015; 30: 1117-1125

47 Jamsa T, Jalovaara P, Peng Z, Vaananen HK, Tuukkanen J. Comparison of three-point bending test and peripheral quantitative computed tomography analysis in the evaluation of the strength of mouse femur and tibia. Bone 1998; 23: 155-161

48 Bagi CM, Hanson N, Andresen C, Pero R, Lariviere R, Turner CH, Laib A The use of micro-CT to evaluate cortical bone geometry and strength in nude rats: correlation with mechanical testing, pOCT and DXA. Bone 2006; 38: 136-144

49 Mittra E, Rubin C, Gruber B, Qin YX. Evaluation of trabecular mechanical and microstructural properties in human calcaneal bone of advanced age using mechanical testing, $\mu \mathrm{CT}$, and DXA. J Biomech 2008; 41: 368-375

50 Wronski TJ, Lowry PL, Walsh CC, Ignaszewski LA. Skeletal alterations in ovariectomized rats. Calcif Tissue Int 1985; 37: 324-328

51 Peng Z, Tuukkanen J, Zhang H, Jämsä T, Väänänen HK. The mechanical strength of bone in different rat models of experimental osteoporosis. Bone 1994; 15: 523-532

52 El Khassawna T, Böcker W, Govindarajan P, Schliefke N, Hürter B, Kampschulte M, Schlewitz G, Alt V, Lips KS, Faulenbach M, Möllmann H, Zahner $D$, Dürselen L, Ignatius A, Bauer N, Wenisch S, Langheinrich AC, Schnettler $R$, Heiss $C$. Effects of multi-deficiencies-diet on bone parameters of peripheral bone in ovariectomized mature rat. PLoS One 2013; 8: e71665 